\title{
INTERVAL ESTIMATES OF FUNCTIONALS IN TIME-DELAY SYSTEMS WITH UNCERTAINTY
}

\author{
YURY V. ZAIKA
}

Received 25 March 2002

\begin{abstract}
We consider a linear dynamical system with delay and uncertainty in initial data and movement and measurement equations. We present an algorithm of estimating an interval of possible values of functionals on solutions. We construct suboptimal weight functions in integral observation operators to minimize a sure estimation.
\end{abstract}

2000 Mathematics Subject Classification: 93C41, 93B07, 93B05.

1. Introduction and problem statement. Theory of time-delay systems with uncertainties has a wide bibliographies. We cite only some papers which contain more detailed bibliographies [1, 2, 3, 5, 6, 7, 9, 10]. Due to applied problems of observation and estimation theory in the infinite-dimensional case, only limited (finite-dimensional) information is known about the phase state or the trajectory, and the values of other given functionals are to be determined. With present uncertainties, it is natural to look for intervals of possible values. By choosing the weight functions for measurements processing, it is useful to minimize the error estimation. This paper may be considered as the realization of common ideas of numerical analysis for the considered problem.

Let the functional differential equations

$$
\dot{x}(t)=f\left(t, x(t), x_{t}, u(t), \mu(t)\right), \quad y(t)=g\left(t, x(t), x_{t}, v(t)\right)
$$

model the movement law and accessible information about the motion. Here, $x(t) \in \mathbb{R}^{n}, y(t) \in \mathbb{R}^{m}, t \geq 0, x_{t}=x_{t}(\cdot):[-h, 0] \rightarrow \mathbb{R}^{n}, x_{t}(\theta)=x(t+\theta)$, $\theta \in[-h, 0], u(t)$ is the control, $\mu(t)$ and $v(t)$ are the perturbation of the movement equation and measurements errors. Components $f$ and $g$ are linear bounded functionals. Detailed theory of functional differential equations is presented, for instance, in [4]. Firstly, general ideas of the paper will be discussed. Consider the following problem: construct an algorithm which allows to determine an interval of possible values of a functional $J=J\left(x(s), x_{s}, \mu(\cdot)\right)$ using any accessible measurements $y(\cdot)$. As for $J$, we can use the components of a vector $x(s)$, projections (Fourier coefficients) of elements $x_{s}, \mu(\cdot)$, and so on. Suppose that the initial data $x(0)$ and $x_{0}(\cdot)$, and noises $\mu(\cdot)$ and $\nu(\cdot)$ are unknown, but limited by a priori given ellipsoid. 
Explicit description of a set $\left\{\left(x(s), x_{s}\right)\right\}$ in a general case seems impossible. Restrictions on $x(0), x_{0}(\cdot), \mu(\cdot)$, and $v(\cdot)$ theoretically allow estimating the phase state $\left(x(s), x_{s}\right)$ in a norm, and obtain an estimation of the possible values of the functional $J$. But due to possible "gluing" effect of solutions of time-delay equations, the initial ellipsoid by the time $s$ may "lose a dimension" and such estimation of $J$ will be for sure rough. To store a continuum of values of $y(t)$ is practically impossible; thus we will assume that during the measuring, a signal $y(t)$ goes to integrators, and weightened integral sums $J_{i}$, $1 \leq i \leq \ell$, are accumulated. The problem appears in a general form as estimating possible values of a given functional using known values of others. Such problems, interpolation, quadratures, and so forth are classical in numerical analysis.

Using a technique of conjugate equations, $J$ and $J_{i}$ can be represented explicitly as functionals on the initial data $x(0), x_{0}(\cdot), \mu(\cdot)$, and $v(\cdot)$. This allows finding precise estimations of possible values of $J$. Moreover, such representation is reasonable from the point of view of stability analysis of $J$ and $J_{i}$ with respect to variations of the initial data and the noises. The geometrical consideration of realized values of $J_{i}=\gamma_{i}, 1 \leq i \leq \ell$, is in the fact that an interval of values $J$ is calculated as a "length of run" of a plane, corresponding to $J$, on an intersection of sections of the initial ellipsoid by $\ell$ planes.

For simplicity, some generalizations for the case of considering uncertainty of both the initial data and the noises will be given at the end of the paper. Firstly, a particular case will be considered: when a main perturbation is of uncertainty of the initial data, the noises in the movement equations and the measurements errors are sufficiently small and can be ignored. Here is the model of the typical application case:

$$
\begin{aligned}
& \dot{x}(t)=\sum_{j=0}^{N} A_{j} x\left(t-h_{j}\right)+\int_{-h}^{0} A(\theta) x(t+\theta) d \theta+B u(t), \quad t \geq 0, \\
& x(0)=x^{0}, \quad x(\tau)=x_{0}(\tau), \quad \tau \in[-h, 0), \quad \hat{x}_{0}=\left(x^{0}, x_{0}(\cdot)\right) \in M_{2}, \\
& y(t)=G x(t), \quad \operatorname{rank} G=m<n, \quad 0=h_{0}<h_{1}<\cdots<h_{N}=h .
\end{aligned}
$$

Here, $M_{2}=\mathbb{R}^{n} \times L_{2}^{n}[-h, 0]$, matrices $A_{j}, B$, and $G$ of dimensions $n \times n, n \times n_{1}$, and $m \times n$ are constant, elements of $A(\cdot)$ and components of a given vector function (control) $u(\cdot)$ are piecewise continuous on the considered time segment $\left[0, t_{*}\right]$. Initial data $\hat{x}_{0}$, consisting of a starting point $x^{0}$ and necessary for (1.2) history $x_{0}(\cdot)$, are unknown.

The solutions of (1.2) are understood as almost everywhere on a segment $\left[0, t_{*}\right]$ (rather large compared to $\left.[0, h]\right)$, where $M_{2}=\mathbb{R}^{n} \times L_{2}^{n}, \hat{x}_{t}=\left(x(t), x_{t}\right)=$ $(x(t), x(t+\cdot)) \in M_{2}$, serves as a phase space. This is because the history influences the movements integrally. The dependence on the initial data $x\left(t ; \hat{x}_{0}, 0\right)$ and $\hat{x}_{t}\left(\hat{x}_{0}, 0\right)$ is noted in a standard way. Vectors $x(0)$ and $x_{0}(0)$ are, in a general case, different. A change in values of $x_{0}(\tau)$ on a zero measure set in 
$[-h, 0]$ does not change the movement $x(t), t \geq 0$. The solution $x(t)$ is absolutely continuous on $\left[0, t_{*}\right]$ (moreover, it belongs to $H^{1}\left(\left[0, t_{*}\right], \mathbb{R}^{n}\right)$ ), thus it is convenient to identify a class $x_{t} \in L_{2}\left([-h, 0], \mathbb{R}^{n}\right)$ with its continuous representative on $[-t, 0] \cap[-h, 0]$. Then $x_{t}(0)=x(t)$, for $t>0$.

Fix a natural $r \geq 1$, and consider the problem of determination of all possible values of a given functional (projection $\hat{x}_{r h}$ )

$$
J=p^{0^{\prime}} x(r h)+\int_{-h}^{0} p^{\prime}(\tau) x(r h+\tau) d \tau=\left\langle\hat{p}, \hat{x}_{r h}\right\rangle_{M_{2}}
$$

Here, $r h \leq t_{*}, \hat{p}=\left(p^{0}, p(\cdot)\right) \in M_{2}$. Let the initial phase uncertainty be limited:

$$
\left\langle\hat{x}_{0}, \hat{x}_{0}\right\rangle_{Q}=x^{0^{\prime}} Q^{0} x^{0}+\int_{-h}^{0} x_{0}^{\prime}(\tau) Q(\tau) x_{0}(\tau) d \tau \leq \bar{\kappa}^{2}
$$

The matrix $Q(t)$ is piecewise continuous. Matrices $Q(t)$ and $Q^{0}$ are symmetrical and positively defined. It is not necessary that $Q^{0}=Q(-0)$.

We suggest that while information $y(t)$ arrives on a time segment $[0,(r-$ 1) $h$ ], the following functionals are calculated:

$$
\begin{array}{r}
J_{i}=\sum_{j=1}^{r-1}\left\langle\hat{k}_{i j}, \hat{y}_{i j}\right\rangle=\sum_{j=1}^{r-1}\left(k_{i j}^{0^{\prime}} y(j h)+\int_{-h}^{0} k_{i j}^{\prime}(\tau) y(j h+\tau) d \tau\right) \\
=\sum_{j=1}^{r-1} k_{i j}^{0^{\prime}} y(j h)+\int_{0}^{\rho} K_{i}^{\prime}(\tau) y(\tau) d \tau, \quad \rho=(r-1) h, \\
\hat{y}_{j h}=(y(j h), y(j h+\cdot)), \quad \hat{k}_{i j}=\left(k_{i j}^{0}, k_{i j}(\cdot)\right) \in \widetilde{M}_{2}=\mathbb{R}^{m} \times L_{2}^{m}, \\
K_{i}(j h+\tau)=k_{i j}(\tau), \quad \tau \in[-h, 0], 1 \leq j \leq r-1,1 \leq i \leq \ell .
\end{array}
$$

We detail the problem. We need an algorithm for determining a segment of possible values of $J$ by known available values of observation (measure processing) functionals $J_{i}, 1 \leq i \leq \ell$. The a priori restrictions on unknown initial data $\hat{x}_{0}$ are taken into account. In case $p(\cdot)=0$ (zeros are all named by one symbol) then, varying $p^{0} \in \mathbb{R}^{n}$, one obtains the components (projections) of the position $x(s)$ at time $s=r h$. When $p^{0}=0$, these are the phase state projections $x_{r h}$ in $L_{2}$ (Fourier coefficients). Some vector weight coefficients $k_{i j}^{0}$ and functions $k_{i j}(\cdot)$ may be zero if, for instance, no measurements on appropriate time segment are made or if the measurements made are unreliable. In particular, it is possible that only discrete measurements $y(j h)$ are used, and then all $k_{i j}(\cdot)=0$. Elements of $\hat{k}_{i j} \in \widetilde{M}_{2}$ are determined by the properties of a certain technical device.

2. Necessary functional representations. Values of the functionals $J$ and $J_{i}$ are determined by the unknown initial data $\hat{x}_{0}$. Thus for estimating $J$ by 
the values of $J_{i}$, it is reasonable to obtain representations $J=J\left(\hat{x}_{0}\right)$ and $J_{i}=J_{i}\left(\hat{x}_{0}\right)$. This will allow not only estimating the initial data variation immunity of the functionals, but also obtaining the precise estimations of the possible values of $J$ (in case initial limitations of the problem are considered precise). The appropriate transforms are based on the following natural idea. If a functional $J$ is given, $J=\left\langle\hat{p}, \hat{x}_{r h}\right\rangle_{M_{2}}=\left\langle\hat{p}, \mathscr{P} \hat{x}_{0}\right\rangle_{M_{2}}$, then it is necessary to find an operator $\mathscr{P}$ and "move it to an argument $\hat{p}$," determining a conjugate $\mathscr{P}^{*}$. In a general case, the representations $J_{i}=J_{i}\left(\hat{x}_{r h}\right)$ are impossible because model (1.2) is not integrable when time decreases.

We define for a homogeneous (i.e., when $u(\cdot)=0$ ) dynamical system with the time-delay (1.2) a shift operator and a conjugate to it:

$$
\begin{gathered}
T, T^{*}: M_{2} \longrightarrow M_{2}, \quad T \hat{x}_{0}=\hat{x}_{h}\left(\hat{x}_{0}, 0\right), \\
\langle\hat{a}, T \hat{z}\rangle_{Q}=\left\langle T^{*} \hat{a}, \hat{z}\right\rangle_{Q} \quad \forall \hat{a}, \hat{z} \in M_{2} .
\end{gathered}
$$

A scalar product $\langle\cdot, \cdot\rangle_{Q}$ is defined in (1.6).

Let us find the convenient representation of the operator $T^{*}$. Fix any vector function $V(\cdot) \in H^{1}\left([-h, 0], \mathbb{R}^{n}\right)$, where the components of $V(\cdot)$ are absolutely continuous and their derivatives (they exist almost everywhere in a classical sense) are square integrable. Denote $x(\cdot)=x(\cdot ; \hat{z}, 0)$. The aim of the following transforms is to move the integrals in an inner product $\langle\hat{a}, T \hat{z}\rangle$ from the argument $\hat{z}$ to $\hat{a}$ by integration by parts:

$$
\begin{aligned}
\langle\hat{a}, T \hat{z}\rangle_{Q}= & a^{0^{\prime}} Q^{0} x(h)+\int_{-h}^{0} a^{\prime}(\tau) Q(\tau) x(h+\tau) d \tau \\
& +\int_{-h}^{0} V^{\prime}(\tau)\left\{\dot{x}(h+\tau)-\sum_{j=0}^{N} A_{j} x\left(h-h_{j}+\tau\right)\right. \\
& \left.\quad-\int_{-h}^{0} A(\theta) x(h+\tau+\theta) d \theta\right\} d \tau \\
= & a^{0^{\prime}} Q^{0} x(h)+\int_{0}^{h} a^{\prime}(s-h) Q(s-h) x(s) d s+V^{\prime}(0) x(h) \\
& -V^{\prime}(-h) x(0)-\int_{0}^{h} \dot{V}^{\prime}(s-h) x(s) d s \\
& -\sum_{j=0}^{N} \int_{-h_{j}}^{h-h_{j}} V^{\prime}\left(s-h+h_{j}\right) A_{j} x(s) d s \\
& -\int_{-h}^{0} V^{\prime}(\tau)\left\{\int_{\tau}^{h+\tau} A(s-h-\tau) x(s) d s\right\} d \tau \\
= & V^{\prime}(0) x(h)-V^{\prime}(-h) x(0) \\
& +\int_{0}^{h}\left(a^{\prime}(s-h) Q(s-h)-\dot{V}^{\prime}(s-h)\right) x(s) d s+a^{0^{\prime}} Q^{0} x(h)
\end{aligned}
$$




$$
\begin{aligned}
& -\sum_{j=0}^{N}\left\{\int_{-h_{j}}^{0} V^{\prime}\left(s-h+h_{j}\right) A_{j} x(s) d s\right. \\
& \left.+\int_{0}^{h-h_{j}} V^{\prime}\left(s-h+h_{j}\right) A_{j} x(s) d s\right\} \\
& -\int_{-h}^{0} V^{\prime}(\tau)\left\{\int_{\tau}^{0} A(s-h-\tau) x(s) d s\right\} d \tau \\
& -\int_{-h}^{0} V^{\prime}(\tau)\left\{\int_{0}^{h+\tau} A(s-h-\tau) x(s) d s\right\} d \tau .
\end{aligned}
$$

Redefine $A(\cdot)$ and $V(\cdot)$ as zero outside of $[-h, 0]$ and choose $V(\cdot)$ on $[-h, 0]$ such that in the last equality there are no values of $x(t)$ when $t \in(0, h]$. This demand implies the following equation:

$$
\begin{gathered}
\dot{V}(t)=-\sum_{j=0}^{N} A_{j}^{\prime} V\left(t+h_{j}\right)-\int_{-h}^{0} A^{\prime}(t-\tau) V(\tau) d \tau+Q(t) a(t), \\
t \in[-h, 0], V(0)=-Q^{0} a^{0}, V(\tau)=0, A(\tau)=0, \tau \notin[-h, 0],
\end{gathered}
$$

which is integrable (at least numerically) from right to left on a time segment $[-h, 0]$. Due to the choice of $V(\cdot)$ according to (2.3), we obtain

$$
\begin{aligned}
\langle\hat{a}, T \hat{z}\rangle_{Q}= & -V^{\prime}(-h) x(0)-\sum_{j=0}^{N} \int_{-h}^{0} V^{\prime}\left(s-h+h_{j}\right) A_{j} x(s) d s \\
& -\int_{-h}^{0} V^{\prime}(\tau)\left\{\int_{-h}^{0} A(s-h-\tau) x(s) d s\right\} d \tau=\langle\hat{c}, \hat{z}\rangle_{Q}, \\
c(t)= & -Q^{-1}(t)\left\{\sum_{j=0}^{N} A_{j}^{\prime} V\left(t-h+h_{j}\right)+\int_{-h}^{0} A^{\prime}(t-h-\tau) V(\tau) d \tau\right\},
\end{aligned}
$$

$V(\tau)=0, A(\tau)=0, \tau \notin[-h, 0]$. Thus, by definition,

$$
T^{*} \hat{a}=\hat{c}, \quad \hat{c}=\left(c^{0}, c(\cdot)\right), c^{0}=-Q^{0-1} V(-h) .
$$

The value of the conjugate operator $T^{*}$ on the element $\hat{a} \in M_{2}$ is determined by the solution $V(\cdot)$ of system (2.3), which is called conjugate to (1.2). The representation of $J=J\left(\hat{x}_{0}\right)$ in a homogeneous case (when $u(\cdot)=0$ ) is written in the form

$$
J=\left\langle\hat{p}, \hat{x}_{r h}\right\rangle_{M_{2}}=\left\langle\hat{Q}^{-1} \hat{p}, T^{r} \hat{x}_{0}\right\rangle_{Q}=\left\langle T^{* r} \hat{Q}^{-1} \hat{p}, \hat{x}_{0}\right\rangle_{Q}
$$

Here the following notation is defined: $\hat{Q}^{-1} \hat{p}=\left(Q^{0-1} p^{0}, Q^{-1}(\cdot) p(\cdot)\right) \in M_{2}$. 
Now, using $T^{*}$, transform the functional $J$ on the solutions of the perturbed movement equation (1.2) with $u(\cdot) \neq 0$ :

$$
\begin{aligned}
J= & \left\langle\hat{p}, \hat{x}_{r h}\right\rangle_{M_{2}} \\
= & \left\langle\hat{Q}^{-1} \hat{p}, \hat{x}_{r h}\right\rangle_{Q} \\
= & \left\langle T^{*} \hat{Q}^{-1} \hat{p}, \hat{x}_{(r-1) h}\right\rangle_{Q}-\int_{-h}^{0} V_{r}^{\prime}(\tau) B u(r h+\tau) d \tau \\
= & \left\langle T^{* 2} \hat{Q}^{-1} \hat{p}, \hat{x}_{(r-2) h}\right\rangle_{Q}-\int_{-h}^{0} V_{r}^{\prime}(\tau) B u(r h+\tau) d \tau \\
& -\int_{-h}^{0} V_{r-1}^{\prime}(\tau) B u((r-1) h+\tau) d \tau \\
= & \cdots=\left\langle T^{* r} \hat{Q}^{-1} \hat{p}, \hat{x}_{0}\right\rangle_{Q}-\sum_{j=1}^{r} \int_{-h}^{0} V_{j}^{\prime}(\tau) B u(j h+\tau) d \tau .
\end{aligned}
$$

An appearance of $V_{j}(\cdot)$ at $u(\cdot) \neq 0$ is a consequence of a transform technique $\langle\hat{a}, T \hat{z}\rangle_{Q}$. A vector function $V_{r}(\cdot)$ is determined as the solution of (2.3) which is conjugate to (1.2) with initial data $V(0)=-p^{0}$ and nonhomogeneity $p(t)$ (instead of $Q(t) a(t)$ ). The next $V_{r-1}(\cdot), \ldots, V_{1}(\cdot)$ are determined recurrently (here $\hat{a}=T^{* r-i} \hat{Q}^{-1} \hat{p}$ ):

$$
\begin{aligned}
\dot{V}_{i}(t)= & -\sum_{j=0}^{N} A_{j}^{\prime} V_{i}\left(t+h_{j}\right)-\int_{-h}^{0} A^{\prime}(t-\tau) V_{i}(\tau) d \tau \\
& -\sum_{j=0}^{N} A_{j}^{\prime} V_{i+1}\left(t-h+h_{j}\right)-\int_{-h}^{0} A^{\prime}(t-h-\tau) V_{i+1}(\tau) d \tau,
\end{aligned}
$$

$t \in[-h, 0], V_{i}(0)=V_{i+1}(-h), V_{i}(\tau)=0, A(\tau)=0, \tau \notin[-h, 0]$.

Define on $[0, r h]$ a continuous vector function $b(\cdot)$ by "gluing" $V_{i}(\cdot)$ as follows: $b(i h+\tau)=V_{i}(\tau), \tau \in[-h, 0], i=\overline{1, r}$. Then the following representation is obtained:

$$
\begin{aligned}
J & =\left\langle T^{* r} \hat{Q}^{-1} \hat{p}, \hat{x}_{0}\right\rangle_{Q}-\int_{0}^{r h} b^{\prime}(\tau) B u(\tau) d \tau \\
& =\left\langle T^{* r} \hat{Q}^{-1} \hat{p}, \hat{x}_{0}\right\rangle_{Q}-\left\langle B^{\prime} b, u\right\rangle_{L_{2}} .
\end{aligned}
$$

The sense of such representation is that the functional $J$ is now explicitly presented via the input data $\hat{x}_{0}$ and $u(\cdot)$. Moreover, calculated $T^{* r} \hat{Q}^{-1} \hat{p}$ and $b(\cdot)$ give information about initial data $\hat{x}_{0}$ and control $u(\cdot)$ variations immunity of values $J$. If, for example, the structure of controlling influence (matrix $B$ ) and the vector function $b(\cdot)$ are such that the values $B^{\prime} b(t)$ are negligibly small, 
then the given projection $J$ is naturally called invariant to control $u(\cdot)$. Varying $\hat{p} \in M_{2}(b=b(\hat{p}))$ and making $B^{\prime} b(t) \approx 0$, we obtain a description of the set of such functionals. In the same way, $T^{* r} \hat{Q}^{-1} \hat{p} \approx 0$ is understood.

Transform in the similar way the functionals $J_{i}$ from (1.7):

$$
\begin{gathered}
J_{i}=\sum_{j=1}^{r-1}\left\langle\hat{k}_{i j}, \hat{y}_{j h}\right\rangle=\sum\left\langle G^{\prime} \hat{k}_{i j}, \hat{x}_{j h}\right\rangle=\sum\left\langle\hat{Q}^{-1} G^{\prime} \hat{k}_{i j}, \hat{x}_{j h}\right\rangle_{Q}, \\
\left\langle\hat{k}_{i j}, \hat{y}_{j h}\right\rangle=\left\langle T^{* j} \hat{Q}^{-1} G^{\prime} \hat{k}_{i j}, \hat{x}_{0}\right\rangle_{Q}-\sum_{s=1}^{j} \int_{-h}^{0} V_{i j s}^{\prime}(\tau) B u(s h+\tau) d \tau .
\end{gathered}
$$

Here $P \hat{k} \equiv\left(P k^{0}, P k(\cdot)\right)$. The vector functions $V_{i j j}(\cdot)$ are defined by the conjugate system (2.3) with initial data $V(0)=-G^{\prime} k_{i j}^{0}$ and nonhomogeneity $G^{\prime} k_{i j}(\cdot)$, and $V_{i j j-1}(\cdot), \ldots, V_{i j 1}(\cdot)$ are obtained recurrently by (2.8) (with respect to the third index), that is, in (2.3), $\hat{a}=T^{* j-s} \hat{Q}^{-1} G^{\prime} \hat{k}_{i j}, s=j-1, \ldots, 1$, is assumed.

Defining

$$
\begin{aligned}
& b_{i j}(t)=V_{i j s}(t-s h), \quad t \in[(s-1) h, s h], 1 \leq s \leq j, \\
& b_{i j}(t)=0, \quad t \in(j h, r h], \quad b_{i}(t)=\sum_{j=1}^{r-1} b_{i j}(t), \quad \hat{q}_{i}=\sum_{j=1}^{r-1} T^{* j} \hat{Q}^{-1} G^{\prime} \hat{k}_{i j},
\end{aligned}
$$

we obtain the representation of the measurement processing operators

$$
J_{i}=\left\langle\hat{q}_{i}, \hat{x}_{0}\right\rangle_{Q}-\int_{0}^{r h} b_{i}^{\prime}(\tau) B u(\tau) d \tau=\left\langle\hat{q}_{i}, \hat{x}_{0}\right\rangle_{Q}-\left\langle B^{\prime} b_{i}, u\right\rangle_{L_{2}} .
$$

Here $b_{i}(\tau)=0, \tau \in[(r-1) h, r h]$. By choosing weight elements $\hat{k}_{i j}$, one can affect sensitivity of the functionals $J_{i}$ to variations of $u(\cdot)$ and $\hat{x}_{0}$.

It must be noted that almost all calculations are of the same kind and all call the same subprogram of numerical integration of system (2.3) with a fixed set of initial data and nonhomogeneities.

3. Interval estimations of functional values. So, the functionals $J$ and $J_{i}$ of the problem are represented as

$$
\begin{gathered}
J=\left\langle\hat{q}, \hat{x}_{0}\right\rangle_{Q}-\psi, \quad J_{i}=\left\langle\hat{q}_{i}, \hat{x}_{0}\right\rangle_{Q}-\psi_{i}, \quad \psi=\left\langle B^{\prime} b, u\right\rangle_{L_{2}}, \\
\psi_{i}=\left\langle B^{\prime} b_{i}, u\right\rangle_{L_{2}}, \quad \hat{q}=T^{* r} \hat{Q}^{-1} \hat{p}, \quad \hat{q}_{i}=\sum_{j=1}^{r-1} T^{* j} \hat{Q}^{-1} G^{\prime} \hat{k}_{i j} .
\end{gathered}
$$

The elements $\hat{q}, \hat{q}_{i} \in M_{2}=\mathbb{R}^{n} \times L_{2}^{n}[-h, 0]$ are determined using the definition of the conjugate operator $T^{*}$. Some technical difficulties occur while calculating the vector functions $b$ and $b_{i}$. Later, to understand an estimation algorithm, it is enough to know that $\psi$ and $\psi_{i}$ are constant (zeros in a homogeneous problem $u(\cdot)=0)$. 
We are interested in an algorithm, which allows the estimation of possible values of the functional $J$ by any possible (considering (1.6)) realization $J_{i}=\gamma_{i}$ :

$$
\left|J-\varphi_{1}\left(\gamma_{1}, \ldots, \gamma_{\ell}\right)\right| \leq \varphi_{2}\left(\gamma_{1}, \ldots, \gamma_{\ell}\right) .
$$

As $u(\cdot)$ is known, the functionals

$$
I=J+\psi=\left\langle\hat{q}, \hat{x}_{0}\right\rangle_{Q}, \quad I_{i}=J_{i}+\psi_{i}=\left\langle\hat{q}_{i}, \hat{x}_{0}\right\rangle_{Q}, \quad\left\langle\hat{x}_{0}, \hat{x}_{0}\right\rangle_{Q} \leq \bar{\kappa}^{2}
$$

can be considered instead of $J$ and $J_{i}$. The problem is formulated in terms of functional analysis: to estimate the functional $I$ using the known values $I_{i}$. Among classical problems of calculus, this problem is considered, for instance, in [11].

Imagine a three-dimensional analogue of infinite-dimensional ellipsoid of initial data $\hat{x}_{0}$. The information $I_{i}=\alpha_{i}$ reduces uncertainty in $\hat{x}_{0}$ to the intersection of an ellipsoid by $\ell$ planes. A run of the plane $I=$ const through this intersection gives the set of all possible values of $I$ that we want to find. In auxiliary argumentations, an index $Q$ in scalar products will be omitted as well as a hat sign, $I=\left\langle q, x_{0}\right\rangle, I_{i}=\left\langle q_{i}, x_{0}\right\rangle, \ldots$. The elements $q_{i} \in M_{2}$ are considered linearly independent, otherwise some of the functionals (1.7) carry no additional information about $x_{0}$ and there is no need to calculate them. If $q$ linearly depends on $q_{i}$, then a functional $J$ for all $x_{0} \in M_{2}$ is uniquely determined on the values of $J_{i}$. These are singular cases, so later on a system $q, q_{1}, \ldots, q_{\ell}$ is considered linearly independent.

Here are some ideas based on some well-known geometrical facts. Define in $M_{2}=\mathbb{R}^{n} \times L_{2}^{n}[-h, 0]$ a new scalar product

$$
[z, x]=\left|\begin{array}{cccc}
\langle z, x\rangle & \left\langle z, q_{1}\right\rangle & \ldots & \left\langle z, q_{\ell}\right\rangle \\
\left\langle q_{1}, x\right\rangle & \left\langle q_{1}, q_{1}\right\rangle & \cdots & \left\langle q_{1}, q_{\ell}\right\rangle \\
\vdots & \vdots & \vdots & \vdots \\
\left\langle q_{\ell}, x\right\rangle & \left\langle q_{\ell}, q_{1}\right\rangle & \cdots & \left\langle q_{\ell}, q_{\ell}\right\rangle
\end{array}\right|
$$

Without further corrections, it is not quite an inner product: $[z, z]=0$ implies $z \in \mathscr{L}_{0}, \mathscr{L}_{0}=\mathscr{L}\left\{q_{1}, \ldots, q_{\ell}\right\}$, and $z$ may be nonzero. Here $\mathscr{L}$ is a symbol of a linear hull. To eliminate this difficulty, consider instead a coset space, where $\mathscr{L}_{0}$ will be zero. But later, only Cauchy-Schwarz-Bunyakovskii inequality will be needed:

$$
\begin{aligned}
{[z, x]^{2} } & \leq[z, z] \cdot[x, x]:[z+\lambda x, z+\lambda x] \\
& =\lambda^{2}[x, x]+2 \lambda[z, x]+[z, z] \geq 0 \quad \forall \lambda \in \mathbb{R}^{1},
\end{aligned}
$$

implying that a discriminant $[z, x]^{2}-[z, z] \cdot[x, x]$ is nonpositive. 
The quantity $[z, z]$ is a determinant of the Gram matrix $\Gamma\left\{z, q_{1}, \ldots, q_{\ell}\right\}$. By definition, an element $\Gamma_{i j}\left\{d_{1}, \ldots, d_{s}\right\}=\left\langle d_{i}, d_{j}\right\rangle$. It is known that a linear dependence of $d_{i}$ is the same as $\operatorname{det} \Gamma\left\{d_{1}, \ldots, d_{s}\right\}=0$. Gram matrix is symmetrical and nonnegatively defined. Moreover, it is positively defined if $d_{i}$ are linearly independent. Thus, for $[z, z]$, all axioms of a scalar product hold, except only $[z, z]=0$ if and only if $z=0$. The last is not important here and, as has already been noted, can be overcome by coset terms.

Write the determinant using an orthogonal expansion:

$$
\begin{gathered}
z=z^{0}+z^{\perp}, z^{0} \in \mathscr{L}_{0}, z^{\perp} \in \mathscr{L}_{0}^{\perp}, \\
\left\langle z^{0}, z^{\perp}\right\rangle=\left\langle q_{i}, z^{\perp}\right\rangle=0 \Longrightarrow[z, z]=\left\langle z^{\perp}, z^{\perp}\right\rangle \operatorname{det} \Gamma_{0}, \quad \Gamma_{0}=\Gamma\left\{q_{1}, \ldots, q_{\ell}\right\} .
\end{gathered}
$$

To do this, represent a row

$$
\left(\langle z, z\rangle, \ldots,\left\langle z, q_{\ell}\right\rangle\right)=\left(\left\langle z^{0}, z^{0}\right\rangle+\left\langle z^{\perp}, z^{\perp}\right\rangle, \ldots,\left\langle z^{0}, q_{\ell}\right\rangle\right)
$$

as a sum $\left(\left\langle z^{0}, z^{0}\right\rangle, \ldots,\left\langle z^{0}, q_{\ell}\right\rangle\right)+\left(\left\langle z^{\perp}, z^{\perp}\right\rangle, \ldots, 0\right)$, and expand the determinant to the sum of two, one of which is $\operatorname{det} \Gamma\left\{z^{0}, q_{1}, \ldots, q_{\ell}\right\}=0$. Thus, the quantity $[z, z] / \operatorname{det} \Gamma_{0}=\left\langle z^{\perp}, z^{\perp}\right\rangle$ is a square of the distance in metrics defined by the scalar product $\langle\cdot, \cdot\rangle=\langle\cdot, \cdot\rangle_{Q}$, from the element $z$ to the linear hull $\mathscr{L}_{0}=$ $\mathscr{L}\left\{q_{1}, \ldots, q_{\ell}\right\}$.

Return to the initial problem. Let the values $I_{i}=\alpha_{i}, i=\overline{1, \ell}$, appear to be known as a result of measuring $y(t)$ and calculating $J_{i}$ according to (1.7). Then, using

$$
\left(\langle z, x\rangle,\left\langle z, q_{1}\right\rangle, \ldots,\left\langle z, q_{\ell}\right\rangle\right)=(\langle z, x\rangle, 0, \ldots, 0)+\left(0,\left\langle z, q_{1}\right\rangle, \ldots,\left\langle z, q_{\ell}\right\rangle\right)
$$

for the first row, after transforming determinants in $\left[\hat{q}, \hat{x}_{0}\right]^{2} \leq[\hat{q}, \hat{q}] \cdot\left[\hat{x}_{0}, \hat{x}_{0}\right]$,

$$
\begin{gathered}
\left|I-I_{*}\right| \leq F_{1} F_{2}, \\
I_{*}=-\frac{\operatorname{det}\left(\begin{array}{cc}
0 & \alpha^{\prime} \\
\sigma & \Gamma_{0}
\end{array}\right)}{\operatorname{det} \Gamma_{0}}, \quad F_{1}^{2}=\frac{\operatorname{det} \Gamma}{\operatorname{det} \Gamma_{0}}, \quad F_{2}^{2}=\left\langle\hat{x}_{0}, \hat{x}_{0}\right\rangle+R_{\alpha}, \\
\Gamma=\Gamma\left\{\hat{q}, \hat{q}_{1}, \ldots, \hat{q}_{l}\right\}, \quad \Gamma_{0}=\Gamma\left\{\hat{q}_{1}, \ldots, \hat{q}_{l}\right\}, \quad R_{\alpha}=\frac{\operatorname{det}\left(\begin{array}{cc}
0 & \alpha^{\prime} \\
\alpha & \Gamma_{0}
\end{array}\right)}{\operatorname{det} \Gamma_{0}}, \\
\alpha=\left(\alpha_{1}, \ldots, \alpha_{l}\right)^{\prime}, \quad \sigma=\left(\left\langle\hat{q}, \hat{q}_{1}\right\rangle, \ldots,\left\langle\hat{q}, \hat{q}_{l}\right\rangle\right)^{\prime}, \quad\langle\cdot, \cdot\rangle=\langle\cdot, \cdot\rangle_{Q} .
\end{gathered}
$$

The geometrical sense of $F_{1}$ is the distance (in a metric, defined by $\langle\cdot, \cdot\rangle_{Q}$ ) from $\hat{q}$ to the linear hull $\mathscr{L}_{0}=\mathscr{L}\left\{\hat{q}_{1}, \ldots, \hat{q}_{\ell}\right\}$ and that of $F_{2}$ is a distance from 
realized $\hat{x}_{0}$ to $\mathscr{L}_{0}$. As $\hat{x}_{0}$ is unknown, then instead of (3.9), an estimation, calculated using $\alpha_{j}$, is obtained:

$$
\left|I-I_{*}\right| \leq F_{1} F_{3}, \quad F_{3}^{2}=\bar{\kappa}^{2}+R_{\alpha} .
$$

Calculating determinants for any realization of $\alpha_{j}$ is not rational, so we find a more convenient interpretation for an estimation. Let

$$
A_{\alpha}=\left\{\hat{x}_{0} \in M_{2} \mid\left\langle\hat{x}_{0}, \hat{x}_{0}\right\rangle_{Q} \leq \bar{\kappa}^{2},\left\langle\hat{q}_{i}, \hat{x}_{0}\right\rangle_{Q}=\alpha_{i}, 1 \leq i \leq \ell\right\} .
$$

Consider, for a fixed $\hat{x}_{0} \in A_{\alpha}$, an orthogonal expansion (note that, in auxiliary calculations, the symbol hat and the index $Q$ are omitted): $x_{0}=x_{*}+v, x_{*} \in \mathscr{L}_{0}$, $v \in \mathscr{L}_{0}^{\perp}$, and $\left\langle x_{*}, v\right\rangle=0$. From $\left\langle q_{i}, x_{0}\right\rangle=\left\langle q_{i}, x_{*}\right\rangle$ and $\left\langle x_{0}, x_{0}\right\rangle=\left\langle x_{*}, x_{*}\right\rangle+$ $\langle v, v\rangle$, it follows that $x_{*} \in A_{\alpha}$. A difference of two elements of $A_{\alpha}$ belongs to $\mathscr{L}_{0}^{\perp}$. Thus, fixing $x_{*}$, we obtain a representation $x_{0}=x_{*}+v$ for all $x_{0} \in A_{\alpha}$. Here a variation $v \in \mathscr{L}_{0}^{\perp}$ is limited by the condition $x_{0} \in A_{\alpha}$. Expansion coefficients $x_{*}=c_{1} q_{1}+\cdots+c_{\ell} q_{\ell}$ due to $\left\langle q_{i}, x_{*}\right\rangle=\alpha_{i}$ are determined from a system of linear equations $\Gamma_{0} c=\alpha$. Thus, $x_{*}$ is determined uniquely, independently of the initially chosen $x_{0} \in A_{\alpha}$, which can also be easily proved for the contraries. If one changes zero in the determinant, which appears in the expression for $I_{*}$, to $\left\langle q, x_{*}\right\rangle-\left\langle q, x_{*}\right\rangle$ and represent the determinant as a sum of two, considering that $x_{*} \in \mathscr{L}_{0}$, then $I_{*}=\left\langle q, x_{*}\right\rangle$. In a similar way $\left(0=\left\langle x_{*}, x_{*}\right\rangle-\left\langle x_{*}, x_{*}\right\rangle\right.$ and $\left.\left\langle q_{i}, x_{*}\right\rangle=\alpha_{i}\right)$, the quantity $F_{2}^{2}=\left\langle x_{0}, x_{0}\right\rangle-\left\langle x_{*}, x_{*}\right\rangle$ is calculated.

Thus, if the coefficient vector $c$ and the element $\hat{x}_{*}=c_{1} \hat{q}_{1}+\cdots+c_{\ell} \hat{q}_{\ell}$ are determined on realized values of $I_{i}=\alpha_{i}$ from the system $\Gamma_{0} c=\alpha$, then the estimation will appear in a more compact form:

$$
\begin{gathered}
\left|I-I_{*}\right| \leq F_{1} F_{2} \leq F_{1} F_{3}, \quad I=\left\langle\hat{q}, \hat{x}_{0}\right\rangle, I_{*}=\left\langle\hat{q}, \hat{x}_{*}\right\rangle, \\
F_{2}^{2}=\left\langle\hat{x}_{0}, \hat{x}_{0}\right\rangle-\left\langle\hat{x}_{*}, \hat{x}_{*}\right\rangle \leq \bar{\kappa}^{2}-\left\langle\hat{x}_{*}, \hat{x}_{*}\right\rangle=F_{3}^{2} .
\end{gathered}
$$

This estimation is precise. It is possible to move in limits of $A_{\alpha}$ from a point $x_{*}$ in direction $v \in \mathscr{L}_{0}^{\perp}$ up to the bound of the ellipsoid. Let $x_{0}=x_{*}+\lambda q^{\perp}$ $\left(q=q^{0}+q^{\perp}, q^{0} \in \mathscr{L}_{0}, q^{\perp} \in \mathscr{L}_{0}^{\perp}\right)$ with a number parameter $\lambda$ from the condition $\left\langle x_{0}, x_{0}\right\rangle=\bar{\kappa}^{2}$. Calculate the quantities which appear in (3.13):

$$
\begin{aligned}
\left|I-I_{*}\right| & =\left|\left\langle q, x_{*}+\lambda q^{\perp}\right\rangle-\left\langle q, x_{*}\right\rangle\right|=|\lambda|\left\langle q^{\perp}, q^{\perp}\right\rangle, \\
F_{1}^{2} & =\operatorname{det} \Gamma / \operatorname{det} \Gamma_{0}=\rho^{2}\left\{q, \mathscr{L}_{0}\right\}=\left\langle q^{\perp}, q^{\perp}\right\rangle, \\
F_{2}^{2}=F_{3}^{2} & =\left\langle x_{*}+q^{\perp}, x_{*}+q^{\perp}\right\rangle-\left\langle x_{*}, x_{*}\right\rangle=\lambda^{2}\left\langle q^{\perp}, q^{\perp}\right\rangle .
\end{aligned}
$$

All inequalities become equalities. We get precisely a segment of possible values of the functional $I$ when $I_{i}=\alpha_{i}, 1 \leq i \leq \ell$. An element $x_{*}$, which defines a centre of the segment $I_{*}=\left\langle q, x_{*}\right\rangle$, is naturally taken as an estimation of the unknown initial state $x_{0}$. 
If a biorthogonal system of elements of the form $d_{j}=\beta_{j 1} q_{1}+\cdots+\beta_{j \ell} q_{\ell}$ $\left(\left\langle d_{i}, q_{j}\right\rangle=\delta_{i j}\right.$ is a Kronecker symbol) is constructed using calculated $q_{i}$, then, for all $\alpha$,

$$
\begin{gathered}
\left\langle q_{i}, x_{*}\right\rangle=\alpha_{i}, \quad x_{*} \in \mathscr{L}\left\{q_{j}\right\}=\mathscr{L}\left\{d_{j}\right\} \Rightarrow x_{*}=\sum_{j=1}^{\ell} \alpha_{j} d_{j}, \\
F_{3}^{2}=\bar{\kappa}^{2}-\sum_{i, j=1}^{\ell} \alpha_{i} \alpha_{j} \xi_{i j}, \quad \xi_{i j}=\left\langle d_{i}, d_{j}\right\rangle, \\
I_{*}=\sum_{j=1}^{\ell} \alpha_{j} \xi_{j}, \quad \xi_{j}=\left\langle q, d_{j}\right\rangle .
\end{gathered}
$$

We now find a more convenient representation of $F_{1}$ (a distance $\rho\left\{q, \mathscr{L}_{0}\right\}$ from $q$ to the linear hull of $q_{j}$ ) using a Fermat theorem for minimization of a positively defined square form with respect to coefficients $\gamma_{i}$ :

$$
\begin{aligned}
& F_{1}^{2}=\min _{\gamma_{i}}\left\|q-\sum_{i=1}^{\ell} \gamma_{i} d_{i}\right\|_{Q}^{2} \Longrightarrow \sum_{i=1}^{\ell} \gamma_{i}^{*}\left\langle d_{i}, d_{j}\right\rangle=\left\langle q, d_{j}\right\rangle, \\
& q-\sum_{i=1}^{\ell} \gamma_{i}^{*} d_{i}=q^{\perp}, \quad\left\langle d_{i}, q_{j}\right\rangle=\delta_{i j} \Longrightarrow \gamma_{i}^{*}=\left\langle q, q_{i}\right\rangle, \\
& F_{1}^{2}=\rho^{2}\left\{q, \mathscr{L}_{0}\right\}=\left\langle q^{\perp}, q^{\perp}\right\rangle=\langle q, q\rangle-\sum_{i=1}^{\ell}\left\langle q, q_{i}\right\rangle\left\langle q, d_{i}\right\rangle .
\end{aligned}
$$

Now we formulate step by step a preliminary algorithm (simplifying the notation):

(1) calculate elements $q$ and $q_{i} \in M_{2}$, vector functions $b(\cdot)$ and $b_{i}(\cdot)$ in the representation of $J\left(x_{0}\right)$ and $J_{i}\left(x_{0}\right)$ in the forms (2.9) and (2.12), and the integrals $\psi$ and $\psi_{i}$ (for a homogenous system $b=b_{i}=\psi=\psi_{i}=0$ ) integrating (2.3) with different $a$;

(2) determine a system of elements $\left\{d_{j}\right\}$ which is biorthogonal to $\left\{q_{i}\right\}$ (this demands to solve a system of linear equations with matrix $\Gamma_{0}$ );

(3) calculate values of $\xi_{i}=\left\langle q, d_{i}\right\rangle$ and $\xi_{i j}=\left\langle d_{i}, d_{j}\right\rangle$ and determine $F_{1}^{2}$ using the obtained above formula.

After these preliminary calculations, it is necessary to "memorize" only the elements $d_{j}$ and the numbers $\psi, \psi_{i}, F_{1}, \xi_{j}$, and $\xi_{i j}$.

Actually, the algorithm which estimates $J$ works as follows. Using the realized values of the functionals $J_{i}$ in (1.7), we calculate $\alpha_{i}=J_{i}+\psi_{i}$ and a square form $F_{3}^{2}$ according to (3.15), and obtain $J_{*}=\alpha_{1} \xi_{1}+\cdots+\alpha_{\ell} \xi_{\ell}-\psi$. A precise estimation of possible values of a functional $J$ is given by the formula $\left|J-J_{*}\right| \leq F_{1} F_{3}$. Here a complete distinctness of $J=J_{*}$ when $F_{2}=0$ is not rejected. An optimal estimating element, if necessary, is defined by a linear combination $x_{*}=\alpha_{1} d_{1}+\cdots+\alpha_{\ell} d_{\ell}$. 
4. Optimization of estimation. Estimation structure gives an idea that to minimize $F_{1}$, it is reasonable to choose one of the functionals (say $J_{1}$ ) according to the condition $\left\|\hat{q}-\hat{q}_{1}\right\|_{Q} \rightarrow$ min. And other functionals $J_{2}, \ldots, J_{\ell}$ should be chosen such that most probable realizations of $\hat{x}_{0}$ are close to $\mathscr{L}_{0}$. If only guaranteed estimations $\left(\left|I-I_{*}\right| \leq F_{1} \bar{\kappa}\right.$, $\left.\max F_{2}=\max F_{3}=\bar{\kappa}\right)$ are considered, then a problem $\left\|\hat{q}-\hat{q}_{1}\right\|_{Q} \rightarrow$ min remains, but a choice of $J_{2}, \ldots, J_{\ell}$ does not influence $F_{1}$ and is not important. Thus, it is interesting to consider the following optimization problem:

$$
\left\|T^{* r} \hat{Q}^{-1} \hat{p}-S \hat{K}\right\|_{Q} \rightarrow \min .
$$

Subject of optimization is a set of elements $\widehat{K}=\left(\hat{k}_{1}, \ldots, \hat{k}_{r-1}\right) \in \widetilde{M}_{2}^{r-1}$, and an operator $S: \widetilde{M}_{2}^{r-1} \rightarrow M_{2}$ is defined by the formula

$$
S \hat{K}=\sum_{j=1}^{r-1} T^{* j} \hat{Q}^{-1} G^{\prime} \hat{k}_{j} .
$$

Problem (4.1) means that we are interested in a quasisolution [8] of the operator equation $S \hat{K}=\hat{q}\left(\hat{q}=T^{* r} \hat{Q}^{-1} \hat{p}\right)$ in the space $\widetilde{M}_{2}^{r-1}$. Here we use in $M_{2}$ a norm generated by the inner product $\langle\cdot, \cdot\rangle_{Q}$.

In a standard way, it is proved (for instance, with the use of a Gronwall lemma) that linear operators $T, T^{*}: M_{2} \rightarrow M_{2}$ are totally continuous. Thus $S \widetilde{M}_{2}^{r-1}$ is not closed. An exception is only if $S$ is finite dimensional, but this is an evidence of the initial model (1.2) being singular. Thus, solvability of the equation $S \hat{K}=\hat{q}$ is not guaranteed. Moreover, a problem (4.1) can have no solution. Here, we meet typical difficulties of solving ill-posed problems (an equation of the first kind with totally continuous operator) [8]. But impossibility in the general case of solving a problem does not mean that it is impossible to find a reasonable approximate solution of (4.1). There is a vast literature about regularization of ill-posed problems. There are no barriers of using them. This way is well studied, but difficult to use. We try to benefit from a specific structure of the operator $S$.

Let $\widehat{B}=\widehat{Q}^{-1} G^{\prime}=\left(Q^{0^{-1}} G^{\prime}, Q^{-1}(\cdot) G^{\prime}\right)$ and note that

$$
\begin{aligned}
S \hat{K} & =\sum_{j=1}^{r-1} T^{* j} \hat{Q}^{-1} G^{\prime} \hat{k}_{j} \\
& =T^{*}\left(\hat{B} \hat{k}_{1}+\cdots+T^{*}\left(\hat{B} \hat{k}_{r-3}+T^{*}\left(\hat{B} \hat{k}_{r-2}+T^{*}\left(\widehat{B} \hat{k}_{r-1}+0\right)\right)\right) \cdots\right) .
\end{aligned}
$$

Consider a discrete dynamical system in $M_{2}$ :

$$
X_{1}=0, \quad X_{i+1}=T^{*} X_{i}+T^{*} \hat{B} \hat{u}_{i}, \quad \hat{u}_{i} \in \widetilde{M}_{2} .
$$

Define controls $\hat{u}_{1}=\hat{k}_{r-1}, \ldots$ and $\hat{u}_{r-1}=\hat{k}_{1}$. Then $X_{r}=S \hat{K}$ and the problem is now posed in terms of a control theory, choosing the controls $\hat{u}_{i}$ which 
transfer a phase point from zero to $\hat{q}$ by $r$ steps. In the context of a control theory, it is clear why it is impossible in the general case to solve the problem $X_{r}=\hat{q}$ : any system has its own attainability set and it is difficult to achieve a complete controllability in an infinite-dimensional case.

Now note that for (4.4), it is sufficient to study controllability by $r$ steps in the linear variety $T^{* r} M_{2}=\left\{\hat{q}=T^{* r} \hat{Q}^{-1} \hat{p} \mid \hat{p} \in M_{2}\right\}$. Unfortunately, excluding a singular case when $T^{*}$ is finite dimensional, the linear set $T^{* r} M_{2}$ is not a subspace of $M_{2}$. While $r$ grows, not only the attainability set $\left(D_{r+1}(0)=\right.$ $\left.\left\{X_{r+1}\right\} \supseteq D_{r}(0)=\left\{X_{r}\right\}\right)$ extends, but also the goal set $T^{* r} M_{2}$ itself "moves towards" $\left(T^{* r+1} M_{2} \subseteq T^{* r} M_{2}\right)$. Thus the problem $S \hat{K}=\hat{q}$, having no solution when $r$ is fixed, may become solvable when $r$ grows, that is, when the system (1.2) is observed for a longer time. Here appears a problem: at what conditions on $A_{j}, A(\cdot), \hat{Q}$, and $G$ is it possible, with a certain $r$, that an absorption by the growing attainability set $D_{r}(0)$ of the narrowing goal set $T^{* r} M_{2}$ occurs? If $D_{r}(0) \supseteq T^{* r} M_{2}$ holds, then in the obtained estimations it is possible to achieve $F_{1}=0$ and then the values of $J=\left\langle\hat{p}, \hat{x}_{r h}\right\rangle_{M_{2}}$ are defined precisely for all $p \in M_{2}$. But this means that there is a possibility to reconstruct $\hat{x}_{r h}$ uniquely by the measurements (1.4), and thus a movement of a system (1.2) for $t \geq r h$. This property is called a complete observability.

In applications, the great efforts for solving an infinite-dimensional absorption $D_{r}(0) \supseteq T^{* r} M_{2}$ are rarely reasonable, for the model describes approximately a real process. Thus, we return to an estimation problem $J=\left\langle\hat{p}, \hat{x}_{r h}\right\rangle_{M_{2}}$. An estimation of a sufficient number of such functionals (Fourier coefficients) gives an approximation of the phase state $\hat{x}_{r h}$.

Let $r$ and $\hat{p}$ be fixed. Assuming that there is no precise solution to a two-point control problem $X_{1}=0$ and $X_{r}=\hat{q}$ (in this case it is possible to make $F_{1}=0$ and uniquely restore the values of $J=I_{*}-\psi$ with infinite-dimensional uncertainty (1.6) of the initial data $\hat{x}_{0}$ ), we consider the problem $\left\|X_{r}-\hat{q}\right\|_{Q} \rightarrow \min$. But this problem is also ill posed. A set of attainability (4.4) is described as the linear hull $\mathscr{L}\left\{T^{* r-1} \widehat{B} \widetilde{M}_{2}, \ldots, T^{*} \widehat{B} \widetilde{M}_{2}\right\}$. This "sum of rotating-by- $T^{*}$ planes" in an infinite-dimensional case is not closed and the projections $\hat{q}$ on $\mathscr{L}$ (in $M_{2}$ with $\langle\cdot, \cdot\rangle_{Q}$ ), which would determine an optimal $\hat{K}$, may not exist. We construct a suboptimal set $\hat{K}$ of weight elements $\hat{k}_{j}$. The sense of a prefix sub will be detailed later.

A dynamical programming method will be used. Let just before the last control step the system (4.4) be in the state $X_{r-1}^{0}$, which will be considered as an unknown parameter. Optimal $\hat{u}_{r-1}$ must solve the problem

$$
\left\|X_{r}-\hat{q}\right\|_{Q} \rightarrow \min , \quad X_{r}=T^{*} X_{r-1}^{0}+T^{*} \hat{B} \hat{u}_{r-1} .
$$

Refusing the solution (quasisolution) to an ill-posed problem

$$
T^{*} \hat{B} \hat{u}_{r-1}=\hat{q}-T^{*} X_{r-1}^{0}, \quad \hat{B}=\hat{Q}^{-1} G^{\prime},
$$


with a parameter $X_{r-1}^{0}$, consider the similar problem

$$
\left\|X_{r}-\hat{q}\right\|_{Q} \leq\left\|T^{*}\right\| \cdot\left\|X_{r-1}^{0}+\hat{B} \hat{u}_{r-1}-T^{* r-1} \hat{Q}^{-1} \hat{p}\right\|_{Q} \rightarrow \min
$$

Instead of the goal function, its upper bound will be minimized. A new problem is in fact a finite-dimensional least squares problem. It has a unique solution

$$
\hat{u}_{r-1}^{0}\left(X_{r-1}^{0}\right)=\hat{N}\left(T^{* r-1} \hat{Q}^{-1} \hat{p}-X_{r-1}^{0}\right), \quad \hat{N}=\left(G \hat{Q}^{-1} G^{\prime}\right)^{-1} G
$$

Obtaining a formula (4.8) is easy, if one remembers a standard problem in $\mathbb{R}^{m}$ : $f(x)=\|A x-b\|_{\mathbb{R}^{n}} \rightarrow \min$, where $A=A_{n \times m}, \operatorname{rank} A=m \leq n$. Expand $f^{2}(x)$ : $\langle A x-b, A x-b\rangle_{\mathbb{R}^{n}}=\left\langle A^{\prime} A x, x\right\rangle-2\langle A x, b\rangle+\langle b, b\rangle$. A matrix $A^{\prime} A$, as a Gram matrix of linearly independent columns of $A$, is symmetrical and positively defined. Thus, a positively defined square form of $x$ is obtained. A minimum is unique and is determined using a Fermat theorem: $2 A^{\prime} A x^{0}-2 A^{\prime} b=0, x^{0}=$ $\left(A^{\prime} A\right)^{-1} A^{\prime} b$.

Remember that according to accepted notation, short expressions with "hats" are understood by context: for all $\hat{a}=\left(a^{0}, a(\cdot)\right) \in M_{2}$,

$$
\begin{aligned}
\left(G \hat{Q}^{-1} G^{\prime}\right)^{-1} G \hat{a} & =\left(G Q^{0-1} G^{\prime}, G Q^{-1}(\cdot) G^{\prime}\right)^{-1} G \hat{a} \\
& =\left(\left(G Q^{0-1} G^{\prime}\right)^{-1} G a^{0},\left(G Q^{-1}(\cdot) G^{\prime}\right)^{-1} G a(\cdot)\right) \in \widetilde{M}_{2}
\end{aligned}
$$

Note that $\widehat{u}_{r-1}^{0}$ is found as the function $\hat{u}_{r-1}^{0}\left(X_{r-1}^{0}\right)$ of an a priori unknown initial state (4.4) on the last step.

After substituting (4.8) in (4.7), we obtain an optimal value

$$
\begin{gathered}
\left\|T^{*}\right\| \cdot\left\|X_{r-1}^{0}+\widehat{B} \hat{u}_{r-1}^{0}\left(X_{r-1}^{0}\right)-T^{* r-1} \hat{Q}^{-1} \hat{p}\right\|_{Q} \\
=\left\|T^{*}\right\| \cdot\left\|\widehat{M} X_{r-1}^{0}-\widehat{M} T^{* r-1} \widehat{Q}^{-1} \hat{p}\right\|_{Q}, \\
\widehat{M}=\hat{E}-\hat{B} \hat{N}, \hat{E}=\left(E_{n}, E_{n}\right)\left(\hat{E} \hat{a}=\hat{a} \in M_{2}\right) .
\end{gathered}
$$

Direct check proves that $\widehat{M}^{2}=\widehat{M},(\widehat{B} \widehat{N})^{2}=\widehat{B} \widehat{N}$,

$$
\langle\widehat{M} \hat{a}, \hat{a}\rangle_{Q}=\langle\hat{a}, \widehat{M} \hat{a}\rangle_{Q}, \quad\langle\hat{B} \hat{N} \hat{a}, \hat{a}\rangle_{Q}=\langle\hat{a}, \hat{B} \hat{N} \hat{a}\rangle_{Q}
$$

That is, $\widehat{M}, \hat{B} \widehat{N}: M_{2} \rightarrow M_{2}$ are in terms of a scalar product $\langle\cdot, \cdot\rangle_{Q}$ the operators of orthogonal projection (orthoprojectors). The result of the influence of $\hat{B} \hat{N}$ on the element $\hat{a} \in M$ is an orthogonal projection of $\hat{a}$ to $\widehat{B} \widetilde{M}_{2}$, and $\widehat{M}$ orthogonally projects on $\left(\widehat{B} \widetilde{M}_{2}\right)^{\perp} \subset M_{2}$. Norms of $\widehat{M}$ and $\hat{B} \hat{N}$ as operators are equal to 1 . Indeed, the projection is not longer than a projected element, and on the projecting subspace, this operator is unit. A right part of (4.10) can be 
estimated now by the inequality

$$
\begin{aligned}
& \left\|T^{*}\right\| \cdot\left\|X_{r-1}^{0}-T^{* r-1} \hat{Q}^{-1} \hat{p}\right\|_{Q} \\
& \quad=\left\|T^{*}\right\| \cdot\left\|T^{*} X_{r-2}^{0}+T^{*} \hat{B} \hat{u}_{r-2}^{0}-T^{* r-1} \hat{Q}^{-1} \hat{p}\right\|_{Q} \\
& \quad \leq\left\|T^{*}\right\|^{2} \cdot\left\|X_{r-2}^{0}+\hat{B} \hat{u}_{r-2}^{0}-T^{* r-2} \hat{Q}^{-1} \hat{p}\right\|_{Q} .
\end{aligned}
$$

In the same way, as with (4.7) and (4.8), the last estimation can be optimized:

$$
\widehat{u}_{r-2}^{0}\left(X_{r-2}^{0}\right)=\hat{N}\left(T^{* r-2} \hat{Q}^{-1} \hat{p}-X_{r-2}^{0}\right) .
$$

Continuing this process, we obtain $\hat{u}_{i}^{0}\left(X_{i}^{0}\right)=\hat{N}\left(T^{* i} \hat{Q}^{-1} \hat{p}-X_{i}^{0}\right)$.

Now start moving backward. An initial state $X_{1}^{0}$ is known $\left(X_{1}^{0}=0\right)$. Thus, $\hat{u}_{1}^{0}=$ $\hat{N} T^{*} \hat{Q}^{-1} \hat{p}$, and due to the dynamics of (4.4), $X_{2}^{0}=T^{*} \hat{B} \hat{N} T^{*} \hat{Q}^{-1} \hat{p}$. Substituting a value $X_{2}^{0}$ in $\hat{u}_{2}^{0}\left(X_{2}^{0}\right)$, we get a representation $\widehat{u}_{2}^{0}=\hat{N} T^{*} \widehat{M} T^{*} \hat{Q}^{-1} \hat{p}$, and so on. Finally,

$$
\begin{gathered}
X_{1}^{0}=0, \quad X_{j}^{0}=T^{* j} \widehat{s}-T^{*}\left(\widehat{M} T^{*}\right)^{j-1} \widehat{s}, \quad \widehat{s}=T^{*} \hat{Q}^{-1} \hat{p}, 2 \leq j \leq r, \\
\hat{u}_{1}^{0}=\hat{k}_{r-1}^{0}=\widehat{N} T^{*} \hat{s}, \quad \hat{u}_{j}^{0}=\widehat{k}_{r-j}^{0}=\hat{N} T^{*}\left(\widehat{M} T^{*}\right)^{j-1} \widehat{s}, \quad 2 \leq j \leq r-1 .
\end{gathered}
$$

Thus, an optimization strategy on each estimation step allows getting an explicit solution. An operation $T^{*}$ on the base of integrating a conjugate system (2.3) with different $\hat{a}$ is considered relatively simple. This strategy is approximate (suboptimal). An optimality criteria is as follows:

$$
\Delta^{0}=\left\|\hat{q}-X_{r}^{0}\right\|_{Q}=\left\|T^{*}\left(\widehat{M} T^{*}\right)^{r-1} \widehat{s}\right\|_{Q} .
$$

If $\Delta_{0}=0$, then the values of $J=\left\langle\hat{p}, \hat{x}_{r h}\right\rangle_{M_{2}}$, while using $J_{1}, \hat{k}_{j}=\hat{k}_{j}^{0}$, are precisely determined $\left(F_{1}=0, J=I_{*}-\psi\right.$, and $\left.\ell=1\right)$.

5. Some generalizations. (1) Assume that the movement equations are given in more general terms of a Stieltjes integral with a matrix function $\Phi(\theta)(\Phi(\theta)=$ $\Phi(\theta-0)$ on $(-h, 0))$. The elements of $\Phi$ are of bounded variation on the segment $[-h, 0]$. Then the conjugate system (2.3) appears as

$$
\begin{gathered}
\dot{V}(t)=\int_{-h}^{0}\left[d \Phi^{\prime}(\theta)\right] V(t-\theta)+Q(t) a(t), \quad t \in[-h, 0], \\
V(0)=-Q^{0} a^{0}, \quad V(s)=0, s \notin[-h, 0],
\end{gathered}
$$

where

$$
\Phi(\theta)= \begin{cases}\Phi(-h), & \theta \leq-h \\ 0, & \theta \geq 0\end{cases}
$$


For (1.2),

$$
\Phi(\theta)=-\sum_{j=0}^{N} X_{\left(-\infty,-h_{j}\right]} A_{j}-\int_{\theta}^{0} A(\tau) d \tau, \quad A(s)=0, \quad s \notin[-h, 0] .
$$

Here, $x$ is a characteristic function of a set.

(2) It is possible to add to the right parts of (1.2) and (1.4) the terms $D \mu(t)$ and $N v(t)$, respectively, $\left(D, N\right.$ are the matrices $\left.n \times n_{2}, m \times m_{1}\right)$, which will describe perturbations in movement equations as well as measurement errors. Together with the initial data, noises are unknown, but restricted. So, $\mu^{\prime}(t) \mu(t) \leq \bar{\mu}^{2}, v^{\prime}(t) v(t) \leq \bar{v}^{2},\|\mu\|_{L_{2}} \leq \mu^{+}$, and $\|v\|_{L_{2}} \leq v^{+}$. Instead of a projection (1.5), a more general functional

$$
J=\left\langle\hat{p}, \hat{x}_{r h}\right\rangle_{M_{2}}+\langle w, \mu\rangle_{L_{2}}, \quad L_{2}=L_{2}\left([0, r h], \mathbb{R}^{n_{2}}\right)
$$

will be estimated. Then, instead of the element $\hat{a}$, it is necessary to consider a set of three elements: $z=\left(\hat{x}_{0}, \mu, \hat{v}\right)$, and represent $J=J(z)$ and $J_{i}=J_{i}(z)$ as

$$
\begin{gathered}
J=\left\langle T^{* r} \hat{Q}^{-1} \hat{p}, \hat{x}_{0}\right\rangle_{Q}-\left\langle B^{\prime} b, u\right\rangle_{L_{2}}-\left\langle D^{\prime} b-w, \mu\right\rangle_{L_{2}}, \\
J_{i}=\left\langle\hat{q}_{i}, \hat{x}_{0}\right\rangle_{Q}-\left\langle B^{\prime} b_{i}, u\right\rangle_{L_{2}}+\left\langle\Lambda_{i}, \hat{v}\right\rangle, \quad b_{i}(\tau)=0, \quad \tau \in((r-1) h, r h], \\
\Lambda_{i}=\left(N^{\prime} \hat{k}_{i 1}, \ldots, N^{\prime} \hat{k}_{i r-1}\right), \quad \hat{v}=\left(\hat{v}_{h}, \ldots, \hat{v}_{(r-1) h}\right), \\
\hat{v}_{j h}=(v(j h), v(j h+\cdot)) \in \widetilde{M}_{2},
\end{gathered}
$$

where $\widetilde{M}_{2}=\mathbb{R}^{m_{1}} \times L_{2}\left([-h, 0], \mathbb{R}^{m_{1}}\right)$. Concrete definitions of scalar products is obtained from context. The calculation of $\hat{q}=T^{* r} \hat{Q}^{-1} \hat{p}, \hat{q}_{i}, b(\cdot)$, and $b_{i}(\cdot)$ gives information about how the functionals are immune to noises. For instance, if $D b(t) \approx w(t)$, then $J$ is invariant to perturbations of movement. Varying $\hat{p}$ and letting $w=D b$, we obtain a class of invariant functionals. In a Hilbert space $H=M_{2} \times L_{2} \times\left(\widetilde{M}_{2}\right)^{r-1}$, the following representations hold:

$J=\langle g, z\rangle_{H}-\psi, \quad J_{i}=\left\langle g_{i}, z\right\rangle_{H}-\psi_{i}, \quad g=\left(\hat{q}, w-D^{\prime} b, 0\right), g_{i}=\left(\hat{q}_{i},-D^{\prime} b_{i}, \Lambda_{i}\right)$.

How to estimate a functional $I=\langle g, z\rangle$ by the values $I_{i}=\left\langle g_{i}, z\right\rangle=\alpha_{i}$ is said above (vector function $u$ is given, thus $\psi$ and $\psi_{i}$ are also known).

(3) We consider a situation when a level of noises is low and is compared to uncertainty of initial data. In this case, it is reasonable to realize an algorithm not in extended phase space $H$, but in original $M_{2}$. The calculated $\hat{q}$, $b(\cdot)$ and limitations of the noises allow representing a functional $I$ in the form $I=\left\langle\hat{q}, \hat{x}_{0}\right\rangle+\sigma,|\sigma| \leq \bar{\sigma}, \bar{\sigma}=\left\|w-D^{\prime} b\right\| \mu^{+},\langle\cdot, \cdot\rangle=\langle\cdot, \cdot\rangle_{Q}$. In the same way, $I_{i}=\left\langle\hat{q}_{i}, \hat{x}_{0}\right\rangle+\sigma_{i},\left|\sigma_{i}\right| \leq \bar{\sigma}_{i}$. Assuming then that $\bar{\sigma}$ and $\bar{\sigma}_{i}$ are relatively small, consider the problem of estimating the possible values of a functional $\left\langle q, \hat{x}_{0}\right\rangle$ 
in $M_{2}$ using the values $\left\langle q_{i}, \hat{x}_{0}\right\rangle$, known up to $\bar{\sigma}_{i}$, and considering a restriction $\left\langle\hat{x}_{0}, \hat{x}_{0}\right\rangle \leq \bar{\kappa}^{2}$. Instead of precise values $\beta_{i}=\left\langle\hat{q}_{i}, \hat{x}_{0}\right\rangle$, it is necessary to use known numbers $\alpha_{i}=J_{i}+\psi_{i}=\beta_{i}+\sigma_{i}$ and an error estimation $\left|\sigma_{i}\right| \leq \bar{\sigma}_{i}$. Instead of

$$
z_{*}=\sum_{j=1}^{\ell}\left(\alpha_{j}-\sigma_{j}\right) z_{j}, \quad J_{*}=\sum_{j=1}^{\ell}\left(\alpha_{j}-\sigma_{j}\right) \xi_{j}+\sigma-\psi
$$

$\left(z_{j} \in M_{2},\left\langle\hat{q}_{i}, z_{j}\right\rangle=\delta_{i j}, \xi_{j}=\left\langle\hat{q}, z_{j}\right\rangle, \xi_{i j}=\left\langle z_{i}, z_{j}\right\rangle\right)$ which are not known precisely, it is reasonable to use $\tilde{z_{*}}=z_{*} \in M_{2}, \tilde{J_{*}}=J_{*}$ when $\sigma=\sigma_{j}=0, j=\overline{1, \ell}$.

Calculating realized $J_{i}$ and the quantities $\alpha_{j}=J_{j}+\psi_{j}$, we obtain

$$
J \leq \tilde{J_{*}}+\bar{\sigma}+\max _{\left|\sigma_{j}\right| \leq \tilde{\sigma}_{j}}\left[-\sum_{j=1}^{\ell} \sigma_{j} \xi_{j}+F_{1}\left(\bar{\kappa}^{2}-\sum_{i, j=1}^{\ell}\left(\alpha_{i}-\sigma_{i}\right)\left(\alpha_{j}-\sigma_{j}\right) \xi_{i j}\right)^{1 / 2}\right],
$$

or (more roughly)

$$
J \leq \tilde{J_{*}}+\bar{\sigma}+\sum_{j=1}^{\ell}\left|\xi_{j}\right| \bar{\sigma}_{j}+F_{1}\left(\bar{\kappa}^{2}-\min _{\left|\sigma_{j}\right| \leq \bar{\sigma}_{j}} \sum_{i, j=1}^{\ell}\left(\alpha_{i}-\sigma_{i}\right)\left(\alpha_{j}-\sigma_{j}\right) \xi_{i j}\right)^{1 / 2} .
$$

A minimum of a square function on a parallelepiped can be found using numerical methods. In the same way, a lower bound of $J$ is obtained. It is possible to make simplifications without calculating $z_{j} \in M_{2}$ (like above). In a case when only measurements errors are relatively small, one should in the given way use the elements $g=\left\{\hat{q}, w-D^{\prime} b\right\}, g_{i}=\left\{\hat{q}_{i},-D^{\prime} b_{i}\right\} \in M_{2} \times L_{2}$.

(4) It is possible to formulate the problem in probabilistic terms. Let $x^{0}$ be a random vector, and let $x_{0}(\tau), \mu(t)$, and $\nu(t)$ be vector random processes with square integrable realizations (on the appropriate time segments). Then functionals $J$ and $J_{i}$ are random variables. Let some values of $J_{i}=\gamma_{i}, i=\overline{1, \ell}$, be realized. The given technique allows obtaining a mean squares estimation of the form $E\left(J-f_{1}\left(\gamma_{1}, \ldots, \gamma_{\ell}\right)\right)^{2} \leq f_{2}\left(\gamma_{1}, \ldots, \gamma_{\ell}\right)$ (here $E$ is for mean value) on any possible sample $\gamma_{i}$. If $f_{2}$ is sufficiently small, then it is possible to identify in terms of mean squares metrics a random variable $J$. To obtain an estimation, it is enough to have statistical information about random elements of the model $E(z, z)_{H} \leq \bar{\varphi}^{2}$ :

$$
E\left(J-J_{*}\right)^{2} \leq F_{1}^{2} F_{2}^{2},
$$

where

$$
f_{1}=J_{*}=I_{*}-\psi, \quad f_{2}=F_{1}^{2} F_{2}^{2} .
$$




\section{REFERENCES}

[1] M. Boutayeb, Observers design for linear time-delay systems, Systems Control Lett. 44 (2001), no. 2, 103-109.

[2] B. Feng and Y. Takeuchi, A stage-structured time-delay model of single-species exposed to environmental hormone, Nonlinear Anal. 47 (2001), no. 6, 40594069.

[3] M. García-Sanz, J. C. Guillén, and J. J. Ibarrola, Robust controller design for uncertain systems with variable time delay, Control Engineering Practice $\mathbf{9}$ (2001), no. 9, 961-972.

[4] J. Hale, Theory of Functional Differential Equations, Applied Mathematical Sciences, vol. 3, Springer-Verlag, New York, 1977.

[5] F.-H. Hsiao, J.-D. Hwang, and S.-P. Pan, D-stability analysis for discrete uncertain time-delay systems, Appl. Math. Lett. 11 (1998), no. 2, 109-114.

[6] Y.-P. Huang and K. Zhou, On the robustness of uncertain time-delay systems with structured uncertainties, Systems Control Lett. 41 (2000), no. 5, 367376.

[7] W. Muhammad, O. Meste, and H. Rix, Comparison of single and multiple time delay estimators: application to muscle fiber conduction velocity estimation, Signal Process. 82 (2002), no. 6, 925-940.

[8] A. N. Tikhonov and V. Ya. Arsenin, Solutions of Ill-Posed Problems, Scripta Series in Mathematics, John Wiley \& Sons, New York, 1977.

[9] Z. Wang, B. Huang, and H. Unbehauen, Robust $H_{\infty}$ observer design of linear time-delay systems with parametric uncertainty, Systems Control Lett. 42 (2001), no. 4, 303-312.

[10] L. Yu and J. Chu, An LMI approach to guaranteed cost control of linear uncertain time-delay systems, Automatica J. IFAC 35 (1999), no. 6, 1155-1159.

[11] N. P. Zhidkov, Linear Approximations of Functionals, Izdatel'stvo Moskovskogo Universiteta, Moscow, 1977 (Russian).

Yury V. Zaika: Institute of Applied Mathematical Research, Karelian Research Center, Russian Academy of Sciences, Petrozavodsk 185610, Russia

E-mail address: zaika@krc.kare1ia.ru 


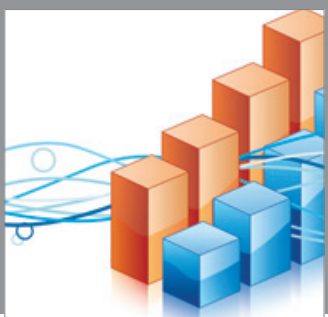

Advances in

Operations Research

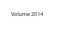

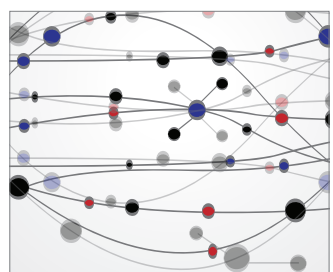

\section{The Scientific} World Journal
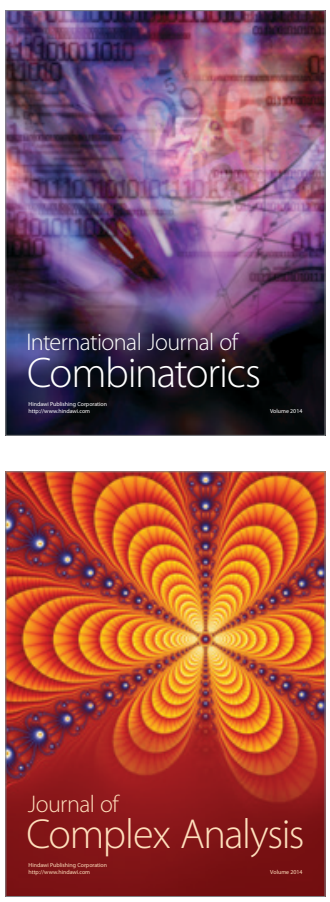

International Journal of

Mathematics and

Mathematical

Sciences
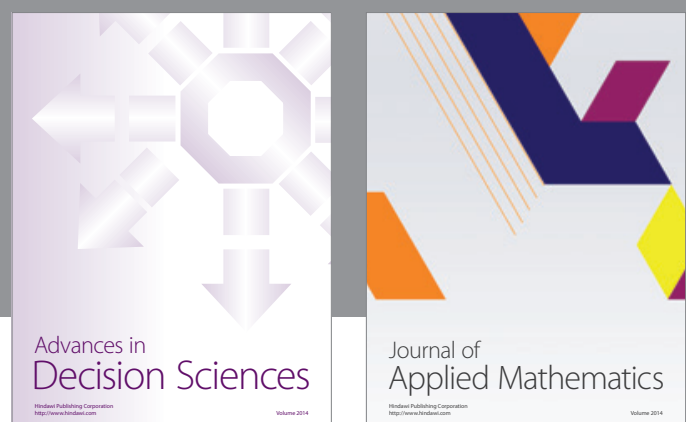

Journal of

Applied Mathematics
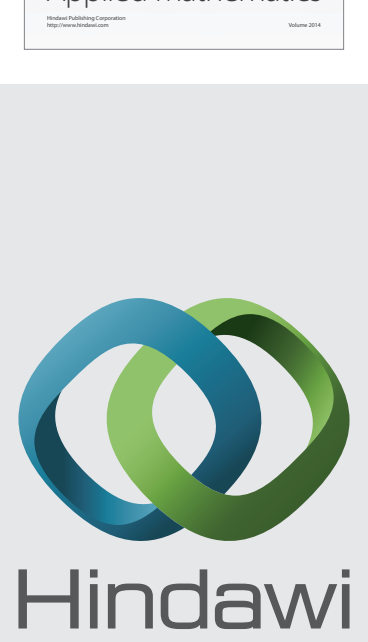

Submit your manuscripts at http://www.hindawi.com
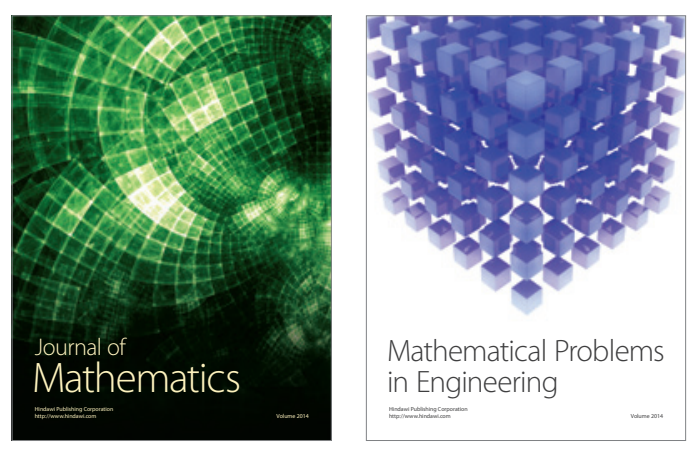

Mathematical Problems in Engineering
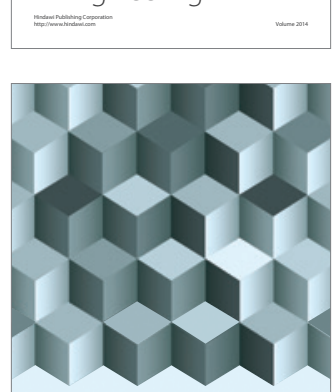

Journal of

Function Spaces
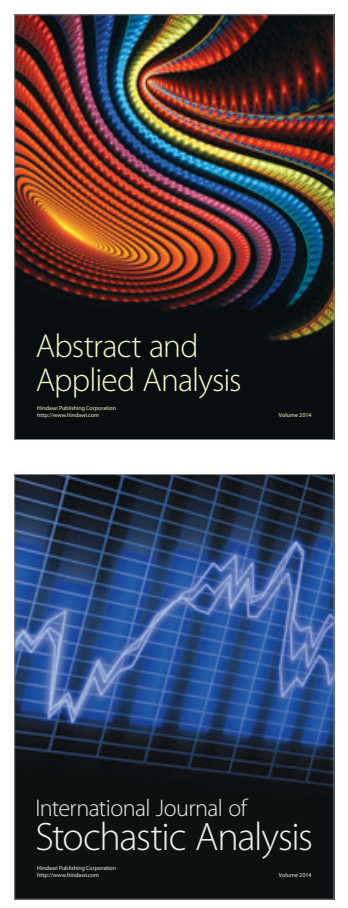

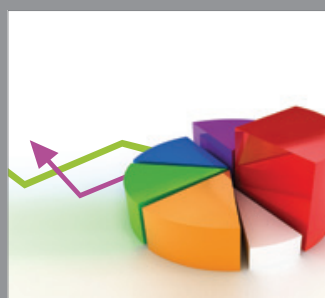

ournal of

Probability and Statistics

Promensencen
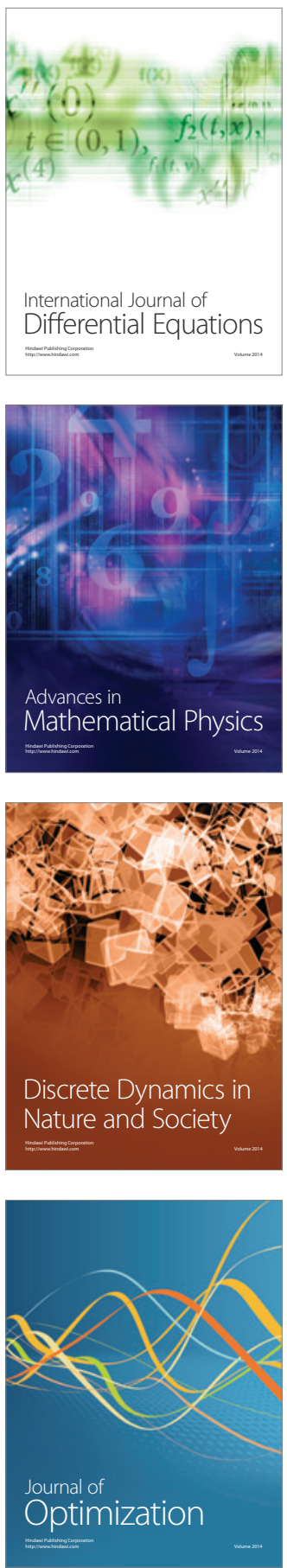